\title{
Examination of Different PAPR Minimization Techniques for MIMO-OFDM System
}

\author{
M. Srinivasa Rao, Sreenievas Alluri, V. Madhav Reddy
}

\begin{abstract}
In this article an analysis on different PAPR minimizing schemes for MIMO-OFDM based wireless communication systems like clipping, selective mapping (SLM) and Partial Transmit Sequence (PTS) are presented. Several properties of OFDM maintain an appealing modulation scheme to support huge data rates in MIMO-OFDM based transmission used in wireless communication system. Apart from several advantages one principle drawback of the OFDM system is it produces huge values of PAPR. The PAPR can be minimized using few schemes like clipping, coding, peak windowing and tone reservation. Simulation results can give the examination between the different PAPR reduction techniques and can give the scope for analyze the different techniques based on different parameters like CCDF values and BER performance with respect to the variation in number sub carriers used for transmission However, huge number of these plans are not able to give a huge degradation in the PAPR by a low computational complexity, but the SLM technique provides essentially better attainment than the other schemes for MIMO-OFDM systems.
\end{abstract}

\section{Keywords: CCDF, MIMO-OFDM and PAPR}

\section{INTRODUCTION}

Presently the multimedia information services have gained more interest and drive us in the $4^{\text {th }}$ generation of wireless communication systems. The need for extensive services in multi-purpose multi reason sight in which there are enormous number of clients with limited range of spectrum, improved the advancements of technologies in digital wireless communication systems have an efficient bandwidth and stable wool-to-channel environment called Multiple Communication System. Current wireless digital communication systems give high information transmission speed for at least many users and are very reliable. In the single carrier system, the whole bandwidth of the system can be utilized by its own carrier, whereas the multi-carrier system has a system bandwidth that spreads from many subcarriers. Therefore, each carrier has a lower transmission bandwidth than the unidirectional transmission bandwidth of a company.

Revised Manuscript Received on December 13, 2019

* Correspondence Author

M. Srinivasa Rao*, Research Scholar, GITAM Institute of Technology, GITAM deemed to be University), Visakhapatnam, India. Email: vasu.srineevas@gmail.com

Dr. Sreenievas Alluri, Associate Professor, GITAM Institute of Technology GITAM (deemed to be University), India. Email: sreenivas.alluri@gitam.edu.in

V. Madhav Reddy, Electronics \& Communication Engineering, Q IS College of Engineering \& Technology, Ongole, India. Email: madhavreddy07@gmail.com
These incredible features of multiple carrier techniques lead us to studying Orthogonal Multimedia Delivering (OFDM).OFDM frame for $4 \mathrm{G}$ wireless communications due to its large constraints on a number of subcarriers, high data rates at abundant $100 \mathrm{Mbps}$ and high portable nearness. OFDM offers a considerable lot of advantages like its heartiness against frequency selective blurring channels and improved spectral efficiency over ISI channels in wireless communication systems; apart from several advantages it has few disadvantages. However the MIMO- OFDM experiences significant downside is that high PAPR of the system.

Several techniques are available for MIMO-OFDM systems to lessening the PAPR, signal distortion techniques like clipping[4] tone injection (TI), tone reservation (TR), and scrambling techniques such as selective mapping (SLM), partial transmit sequence (PTS), active constellation scheme, interleaving and).Signal bending techniques such as clipping and filtering are not suitable, when system comprises of huge number of subcarriers. Remaining other techniques called scrambling and interleaving techniques such as PTS and SLM[4] reduce PAPR in MIMO-OFDM to enormous degree without presenting distortion The difference in the requirement for high data rates is a great deal of attention to the mixed transport framework. It ought to have the option to work smoothly in the atmosphere of a high frequency carrier, high transmission rates and mobility. OFDM has been designed that to meets the needs of the multicarrier modulation systems and shown by research. OFDM is an MCM modular method in different compound information symbols (e.g., BPSK, QPSK, QAM, MPSK, etc.) are altered on the orthogonal subcarrier and then transmitted simultaneously.

One of the main defects of the OFDM system that suffers is from its larger peak power to average power ratio (PAPR) and it causes due to changing the OFDM signal from frequency domain. After converting it to a time domain signal this makes the entire sub carriers are in phase the resultant signal is formed because of accumulation of all sub-carriers. So the result is that the peak power will be $\mathrm{N}$ times larger than the average power. Due to this the OFDM system operation is effected by high PAPR values, driving the amplifier into non linear region of characteristics, thereby distortion occurs in the original information and causes large power will be consumed by an amplifier. 
Orthogonal frequency division multiplexing (OFDM) can be given as an example of multicarrier modulation system in that each and every subcarrier is orthogonal to each other. The time interval of a symbol can be extended by stretching the input bits into N-parallel bit streams because of this modulation scheme. OFDM signals are not affected by multipath propagation because of increased time duration of symbol and features of orthogonality which will improves the spectral efficiency.

One feature of the multi carrier transmission scheme provides high powerful scope for the signal to transmit. The PAPR value of an OFDM signal can be decided by the subcarriers quantity used in the system. Systems comprises of sufficient number of subcarriers can give peripheral probability for the high peaks existence in the system but in practice the number of carrier signals exhibit larger PAPR than with the single carrier signal instance. Multi carrier modulation system that contains the number of Multi carrier signals generates problems in many communication systems; the reason behind is that the power amplifiers at the transmitter very high strength signals. But practically Power amplifiers do not keep up the consistency over the entire effective range of the multiple carrier signals therefore amplification has done inconsistently at disparate chunks of the signal. This offers distortion in the multiple carrier signals and reduces bit error rate (BER) as well as shows growth in spectrum due to the energy radiation at greater frequency values adjacent to the required frequencies of signal when compared with the original spectrum.

Peak power to Average Power proportion [1] in an OFDM signal $\mathrm{s}(t)$ with $\mathrm{N}$ subcarriers in time interval $\tau$ can be expressed as below:

$$
\operatorname{PAPR}[S(t), \tau]=\frac{\max [S(t)]^{2}}{\left.E[[S(t)]]^{2}\right]}
$$

In the above equation (1) $\max [S(t)]^{2}$ indicates the signal peak power value and

$E\left[[S(t)]^{2}\right]$ represents the signal average power.

Among all the techniques available in an OFDM system to reduce the PAPR the simplest method may be considered as this scheme performs the clipping of amplitudes to restrict the peak envelopes in an input signal we assign some threshold value 'for the amplitude. The signals with the values which are higher than this threshold values are removed after that the remaining values are concede to cross through without any disturbance [2]. Therefore high peak problems can be solved by cut-off the peak from the signal using amplitude clipping.

\section{CLIPPING}

In case of clipping the problem arises due to distortion emerges by amplitude clipping and we can treat it as another noise source [5]. In-band and out of band distortions these two distortions are due to this distortion caused by clipping. The PAPR of any reduction technique can be measured in one of the most regularly used parameters is Cumulative
Distribution Function (CDF).Commonly the Complementary $\mathrm{CDF}$ (CCDF) is calculated rather than CDF, it is the measure of probability that PAPR for some data blocks are greater than the threshold value set.

For the signal sample with particular amplitude, the CDF value will be given as

$$
F(z)=1-\exp (z)
$$

Various PAPR reduction techniques and their efficiency can be analyzed by the CCDF estimation for the PAPR for each data block. This is given by

$$
\begin{aligned}
P(P A P R>Z) & =1-P(P A P R \leq Z) \\
& =1-F(z)^{N} \\
& =1-(\exp (Z))^{N}
\end{aligned}
$$

In the above expression we assumed $\mathrm{N}$ samples in time domain and are uncorrelated and mutually independent to each other.

\section{SELECTIVE MAPPING}

Most of these schemes are lamentably not ready to diminish the PAPR to the greater extent with reduced complexity, low coding overhead and with degradation in prominent. Selective mapping can be considered as unique powerful PAPR reduction scheme for OFDM systems among available schemes. This scheme shows that it can accomplish PAPR reduction to several decibels and transmission power efficiency can be significantly improved. However, the major disadvantage of this technique is to send several bits of side information obtain the decoded information blocks at the receiver. Selective mapping technique is an auspicious technique for minimizing PAPR in an OFDM system to a greater extent. The principle operation of Selective Mapping is shown in below fig1.

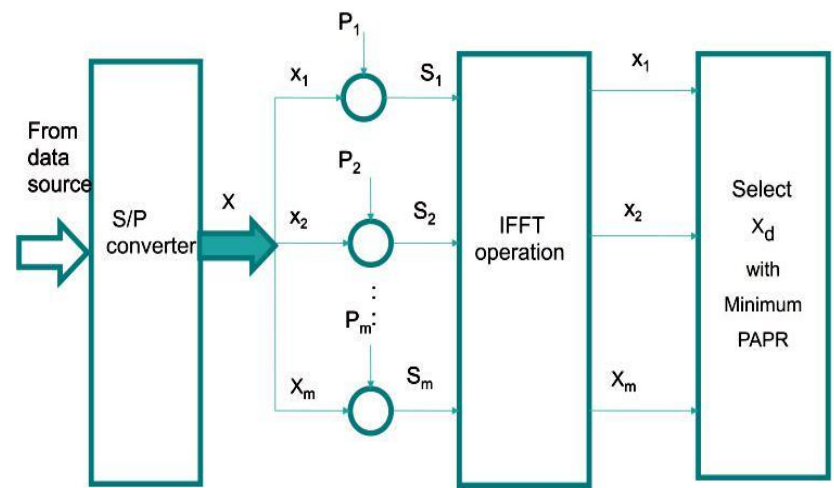

Fig.1. Block Diagram of SLM method

In SLM procedure the fundamental thought is we need to create $\mathrm{M}$ selective candidate signals from the given message block then to choose one of the signal for transmitting out of $\mathrm{M}$ signals which shows the least PAPR [3]. 
This thought produced from the way that the PAPR is gotten from the grouping of vectors to be transmitted $X_{m}$ are multiplied with the information vectors using certain irregular phases these will modify the PAPR values after IFFT operation has been applied. Numerically a square of $M$ uniquely, different, fixed vectors of pseudo random type are formed. Now the original input information $X[X 1$, $\mathrm{X} 2, \ldots, \mathrm{XN}-1]$ and the individual phase sequences $\mathrm{P}=\left[\mathrm{P} 1^{(\mathrm{m})} \mathrm{P}\right.$ $\left.2^{(\mathrm{m})}, \ldots, \mathrm{PN}^{(\mathrm{m})}\right](\mathrm{m}=0,1, \mathrm{M}-1)$ are multiplied where $\mathrm{M}$ indicates the number of phase strings. The $\mathrm{N}$ value is $(\mathrm{m}=0$, $1 \ldots, \mathrm{M}-1)$ is same for both phase sequences and input data Then after multiplication we apply IFFT operation to make the frequency domain signal as time domain signal. This results in the generation of OFDM system that contain message block as different time domain signals with length of $\mathrm{M}$ with distinct values of PAPR,

$\mathrm{X}(\mathrm{m})=\left[\mathrm{X} 1^{(\mathrm{m})}, \mathrm{X} 2^{(\mathrm{m})}, \ldots, \mathrm{X} \quad \mathrm{N}-1^{(\mathrm{m})}\right]$. Then the final step is looking at the PAPR values for each and every individual information blocks and the most minimal PAPR valued candidate signal has to be chosen for transmission [4].

The likelihood that the PAPR about an OFDM signal more than the threshold PAPR value say $z$, can be given by CCDF as below

$$
\begin{aligned}
F z \max (\mathrm{z}) & =\mathrm{P}\left(\mathrm{Z}_{\max }>\mathrm{z}\right) \\
& =1-\mathrm{P}\left(\mathrm{Z}_{\max }<\mathrm{z}\right)=1-F z \max (\mathrm{Z})
\end{aligned}
$$

$$
\mathrm{P}(\mathrm{PAPR}>\mathrm{Z})=\mathrm{F}\left(\mathrm{z}^{\mathrm{N}}=\left(1-\left(1-e^{-z}\right)^{N)^{U}}\right.\right.
$$

Where $\mathrm{M}$ says the number of phase sequences

$N$ gives the number of subcarrier signals

$Z$ is threshold

$Z$ represents any real no

\section{PARTIAL TRANSMIT SEQUENCE}

In PTS technique the approach is to, first block of information segmented as non-overlapping sub-blocks [7]. Later these sub- blocks will be subjected to certain phase change with some factors of rotation and then these block are statistically individual to each other,

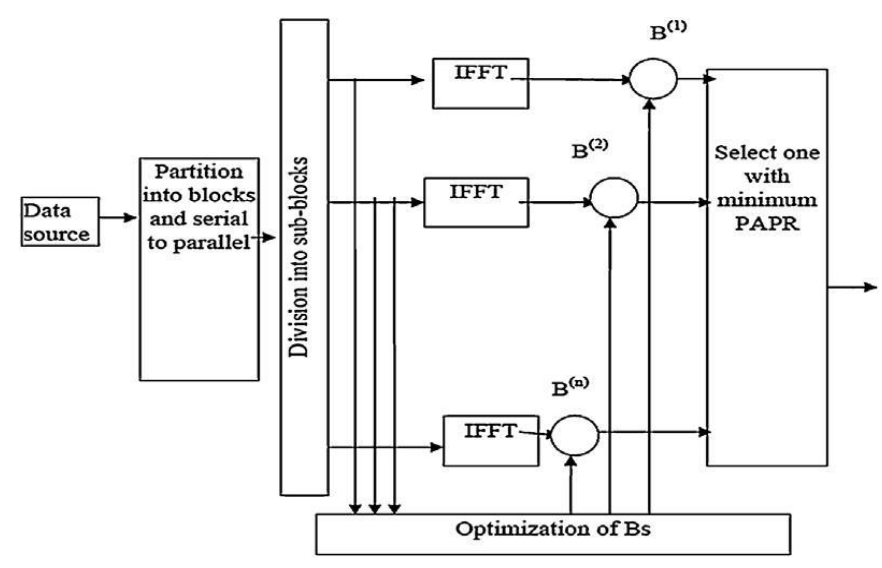

Fig. 2. Block Diagram of PTS technique

\section{RESULT}

Like that the information related to the factor of rotation, and lowest peak amplitude data has been generated in the time domain, then it can be sent to the receiver side. The PTS scheme block diagram has shown in above figure 2
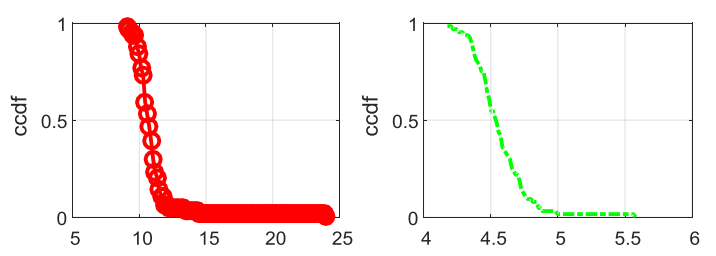

paprO...original signal, $x \mathrm{~dB}$
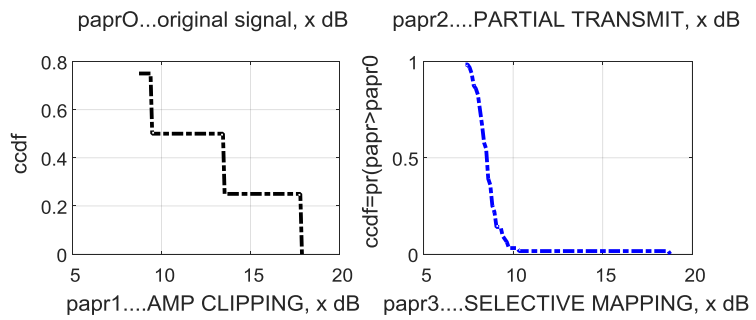

Fig.3. CCDF values for various techniques

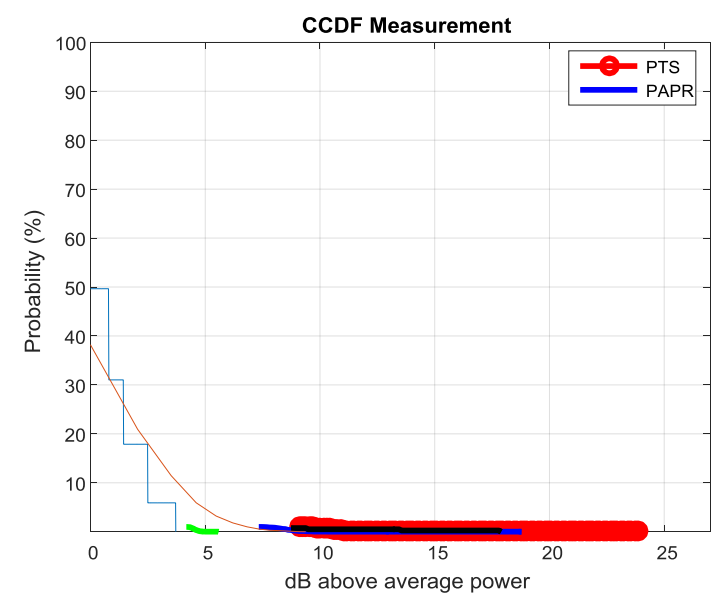

Fig.4. Comparison of CCDF between conventional \&PTS

CCDF values obtained for clipping, PTS and SLM technique represented in graph. From the graphs it can be seen that the PAPR is greatly diminished with the SLM technique but it has the disadvantage that the SI bits needs to be sent along with data bits to perform the extraction of original information bits in the received information. In case of clipping is the PAPR reduction technique, the signals are distorted due to the signals peaks are clipped to reduce PAPR. Hence the particular reduction technique has been applied depends on the particular application.

\section{REFERENCES}

[1] T. Jiang and Wu Y, "An Overview: Peak-to-Average Power Ratio Reduction Techniques for OFDM Signals," Elsevier publication, June 2008, pp.257-268.

[2] G. Ren and et.al "A complementary clipping transform technique for the reduction of peak-to average power ratio of OFDM system," IEEE Trans., pp. 922-926, Nov. 2003.

[3] Lim D W, Seon J, Lim C W "A new SLM OFDM scheme with low complexity for PAPR reduction “ [J]. IEEE Signal,. 2005, 12(2) pp: 93-96. 
[4] P. Cheng, Y. Xiao, L. Dan, S. Li, Improved SLM for PAPR reduction in OFDM system, in: Proc. 2007 IEEE Symp. Personal, Indoor, and Mobile Radio Commun., pp. 1-5.

[5] L. Wang, C. Tellambura , "A simplified clipping and filtering technique for PAR reduction in OFDM systems", IEEE Signal Process. Lett. 12.

[6] Amritpal and Singh, Harjit Singh," Peak to average power ratio reduction in OFDM system using hybrid technique", Elseiver,2015, Optik 127 (2016) 3368-3371.

[7] S. J. Ku, C. L. Wang and C. H. Chen, "A Reduced- Complexity PTS-Based PAPR Reduction Scheme for OFDM Systems," in IEEE Transactions on Wireless Communications, vol. 9, no. 8, pp. 2455-2460, August 2010.

[8] S.H.Leung et. al. "Algorithm for repeated clipping and filtering in peak-to-average power reduction for OFDM" Electronics Letters, $5^{\text {th }}$ December 2002, Vol. 38, No.25, pp.1726-1727

[9] E. Abdullah, A. Idris, and A. Saparon "PAPR Reduction Using SCSSLM Technique in STFBC MIMO-OFDM,” ARPN J. Eng. Appl. Sci., 2017.

[101 JAYALATH, A.D., TELLAMBURA C, "SLM and PTS peak-power reduction of OFDM signals without side information'”, IEEE Trans., 2005 4, pp. 2006-2013

\section{AUTHORS PROFILE}

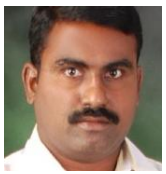

M.Srinivasa Rao received his B.Tech degree in Electronics \&Communication Engineering from JNTUK University, Kakinada, , India in 2006 and M.Tech degree from JNTUK University, Kakinada, India in 2012. He is currently doing his research in the department of Electronics and Communication Engineering of the GITAM Institute of Technology, GITAM (Deemed to be University), Visakapatnam, India. His research area is Machine learning language intelligent based Peak to average power ratio (PAPR) reduction in MIMO- OFDM using Hybrid Technique for high data rate wireless communication system

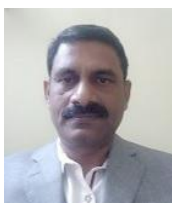

Sreenivas Alluri. received his B.Tech degree in Electronics \& Communication Engineering from University of Kerala, India in 1994, M.Tech in Telecommunication Systems Engineering and Ph.D. in Wireless Communications in 2003 and 2009respectively from Indian Institute of Technology, Kharagpur, India. After various tenures in academia and industries, he joined as a Associate Professor in the Department of Electronics \& Communication Engineering at GITAM Institute of Technology GITAM (Deemed to be University), Visakapatnam, India in 1998 where he is presently working as an Associate Professor. He has published papers in several international conferences and journals, and holds two US patents and three patent applications. Sameer S. M. is a senior member of the IEEE and a regular reviewer of many reputed international conferences and journals. He has served on the Technical Programmes and other Committees of severa International Conferences. His research interests include synchronization and channel estimation techniques for multicarrier communication systems, Wi-MAX system design challenges, optimization techniques for wireless communication, cognitive radio, and physical layer issues in cooperative communication systems. 